\title{
LINHA DO TEMPO INTERATIVA NO ENSINO DE HISTÓRIA
}

\author{
Interactive Timeline in History Teaching
}

\author{
Luis Antonio Tavares* \\ Lucas Lopes Maldonado** \\ Luciane Silva de Almeida ${ }^{* * *}$ \\ Tiago de Morais Pereira ${ }^{* * * *}$ \\ Sérgio Ferreira do Amaral ${ }^{* * * * *}$
}

\begin{abstract}
Resumo: Este artigo apresenta uma proposta de objeto de aprendizagem para a área de conhecimento de história, consistindo em uma linha do tempo interativa na qual o usuário pode navegar por eventos através de sua relação temporal e, inclusive, criar suas próprias linhas do tempo. A proposta tem como contribuição uma aproximação entre as práticas escolares e a realidade tecnológica do cotidiano do estudante, através de uma ferramenta pedagógica interativa. Também é uma solução que busca sanar uma grande dificuldade da era digital: lidar com grandes volumes de dados, possibilitando uma visualização gráfica mais dinâmica e intuitiva. Seu grande diferencial é ser a primeira ferramenta gratuita disponível na internet para criação e estudo usando linhas do tempo interativas.
\end{abstract}

Palavras-chave: Linha do tempo. Aplicações interativas. Tecnologia na educação. Objetos de aprendizagem. Ensino de história.

\begin{abstract}
This article presents a learning object proposal for the history knowledge area, consisting of an interactive timeline in which the user can navigate through events through their temporal relation and even create their own timelines. The proposal has as its contribution an approximation between the school practices and the technological reality of the daily life of the student, through an interactive pedagogical tool. It is also a solution that seeks to solve a great difficulty of the digital age - dealing with large volumes of data, enabling a more dynamic and intuitive graphical visualization. Its great differential is to be the first free tool available on the internet for creation and study using interactive timelines.
\end{abstract}

Keywords: Timeline. Interactive applications. Educational technology. Learning objects. History teaching.

\footnotetext{
* Mestre em Engenharia Elétrica pela FEEC-UNICAMP, Professor no Instituto Federal de Educação, Ciência e Tecnologia do Sul de Minas Gerais - Campus Pouso Alegre, Discente de Doutorado na Faculdade de Educação da UNICAMP, Orcid: https://orcid.org/0000-0001-8076-733X, e-mail: luis.tavares@ifsuldeminas.edu.br

*** Discente do curso de Física do Instituto de Física "Gleb Wataghin" - UNICAMP, Orcid: https://orcid.org/00000002-0166-1893, e-mail: lucasl.maldonado01@gmail.com

**** Doutora em História pela UFMG, Professora no Instituto Federal de Educação, Ciência e Tecnologia de Minas Gerais - Campus Sabará, Orcid: https://orcid.org/0000-0002-7424-1611, e-mail: luciane.almeida@ifmg.edu.br

***** Discente do curso de Engenharia de Computação do Instituto Nacional de Telecomunicações - INATEL, Orcid: https://orcid.org/0000-0001-7892-0980, e-mail: tiagodemoraispereira@gmail.com

****** Professor Doutor da Faculdade de Educação da UNICAMP, Orcid: https://orcid.org/0000-0002-3536-2226, e-mail: amaral@unicamp.br
} 


\section{Introdução}

No mundo atual, vivemos em uma sociedade que busca constantemente novas informações. Neste sentido, o acesso a elas tem se tornado cada dia mais instantâneo, prático e possibilitado por múltiplos dispositivos. Com poucos cliques, é possível ficar por dentro de fatos importantes dos quatro cantos do mundo. Essas informações são essenciais e podem estar relacionadas tanto às notícias do cotidiano quanto a contextos abordados no ensino.

Porém, toda essa quantidade de informação, muitas vezes, não está disponibilizada de modo suficientemente objetivo, claro ou acessível. Em muitos momentos, encontrar nosso ponto de interesse depende de um minucioso filtro num grande universo de dados disponibilizados por múltiplas fontes.

Os serviços da web evoluíram muito para lidar com essa questão, porém ainda há muita demanda para soluções de interface visual. Por exemplo, não é simples observar uma relação temporal através de dados da Wikipedia, e os resultados de uma busca no Google continuam sendo uma lista interminável de links.

Pensando numa maneira de dispor de forma objetiva fatos históricos aos usuários, propomos uma aplicação interativa de linha do tempo. O grande diferencial desta solução é a facilidade de navegação: sua usabilidade. Esta aplicação vem para suprir uma demanda da área de conhecimento de história, área carente por soluções tecnológicas, conforme afirma Freitas (2010).

Atualmente, ao se estudar história, analisar eventos históricos em sua relação temporal pode ser difícil devido à quantidade de tópicos. Portanto, uma solução tecnológica, intuitiva e interativa que permite navegar pelos fatos através de sua relação temporal traz uma gama de contribuições para área, facilitando os estudos.

Ao criar uma solução didática, tecnológica e interativa, também estamos aproximando a realidade da escola à realidade do discente. Muitas vezes a escola se torna uma ilha, com processos de aprendizagem à margem da realidade tecnológica que vivenciamos na nossa sociedade. Nossos discentes estão imersos nesse mundo digital, mais dinâmico e interativo.

A preocupação em aproximar a tecnologia da sala de aula pode ser demonstrada pela Base Nacional Comum Curricular (BNCC) de 2017, a qual destaca na quinta competência geral da educação básica: "Compreender, utilizar e criar tecnologias digitais de informação e comunicação de forma crítica, significativa, reflexiva e ética nas diversas práticas sociais" (BRASIL, 2017).

Neste sentido, é importante ressaltar que, nos Parâmetros Curriculares Nacionais (PCNs) de história, não existe menção ao uso de ferramentas digitais, o aluno ficaria restrito à utilização de livros e alguns documentos. No entanto, a inserção das tecnologias no ensino de história pode começar com a utilização do computador, através de pesquisas em sites via internet, visitas a museus virtuais e consulta a arquivos históricos, e se estender para o uso de aplicativos em celular, tornando as informações mais acessíveis e seu uso mais intuitivo. Este contato certamente possibilitará aos alunos uma compreensão mais ampla de fatos e conceitos que os levem a conhecer o passado, ao passo que constroem uma análise crítica do presente.

Portanto, pensando nesta nova era digital e em contribuir com área de conhecimento de história, bem como em práticas e ferramentas pedagógicas mais tecnológicas e dinâmicas, que instiguem a participação dos discentes, é que ocorre a concepção da proposta deste trabalho. 


\section{Referencial Teórico}

Atualmente as escolas fazem uso de computadores, projetores e outros equipamentos, porém sem uma integração dessas tecnologias numa perspectiva pedagógica, sem material didático e metodologia adequada para somar o potencial das Tecnologias da Informação e Comunicação (TICs) em favor do aprendizado do discente, explicam Cândido et al. (2018).

Do outro lado, temos uma sociedade cada vez mais tecnológica que tem remodelado seus processos sociais pela influência da tecnologia. Segundo Zuin (2010), a tecnologia tratase de um processo social que reconfigura até nossas identidades pessoais. Nessa nova sociedade, os jovens estão acostumados com o dinamismo e a integração possibilitados pelas TICs, na contramão do que encontram na escola.

Para Moran (2007), há uma percepção crescente entre o descompasso dos modelos tradicionais de ensino e as possibilidades que as novas tecnologias oferecem. Moran acrescenta ainda que as tecnologias, além de apoio, são instrumentos fundamentais para a mudança na educação.

A evolução tecnológica criou a necessidade de reestruturação pedagógica e metodológica das práticas de ensino e do cotidiano escolar, portanto urge repensar as formas convencionais de ensino e aprendizagem, aliando a tecnologia ao papel de educador e orientador do professor. Neste sentido, concordamos com Arroyo (2000) quando esclarece que as TICs podem transmitir competências e informações com maior rapidez e eficiência que o professor, no entanto, elas não darão conta do papel socializador da escola, do encontro de gerações e do aprendizado humano que se dá no convívio direto com as pessoas. Assim, é necessário combinar os dois.

A tecnologia é um importante instrumento de aprendizagem. Lévy (1993) confirma essa afirmação quando apresenta a existência de três categorias de conhecimento: a oral, a escrita e a digital, demonstrando que, em nossa sociedade, a ausência de uma dessas categorias limita a inserção social do indivíduo e o seu acesso à cidadania. As categorias escrita e digital, surgidas em tempos completamente diferentes e responsáveis por mudanças determinantes na constituição das sociedades, hoje se unem para promover o conhecimento. Ainda segundo Lévy, essas três formas de apropriação do conhecimento apresentam particularidades no que diz respeito à complexidade das ações, às formas de pensar e de representar a realidade e ao acesso aos meios de inserção social.

Pensando em aproximar a realidade tecnológica às escolas, uma alternativa seria o uso de objetos de aprendizagem (OAs). Tarouco, Fabre e Tassiunas (2003) definem os OAs como qualquer recurso de apoio, suplementar ao processo de aprendizagem, caracterizados como materiais educacionais construídos em pequenos conjuntos com o intuito de maximizar as situações de aprendizagem.

Para Wiley (2001), os OAs são geralmente compreendidos como entidades digitais que podem ser utilizadas em variados contextos de estudo, são caracterizados como tecnologias instrucionais e seu amplo uso no ambiente digital é devido a seus potenciais de reuso, generalização, adaptatividade e escalabilidade. Aguiar e Flores (2014) destacam que os OAs têm a característica de serem autossuficientes em seu contexto de aplicação, não necessitam de complementos, o que marca o potencial de reuso.

Podem ser citados como exemplos de OAs multimídias, páginas web, softwares educacionais, animações, jogos eletrônicos criados para abordar determinado tema. Os OAs 
são, portanto, recursos didáticos adequados para trabalhar com tecnologia em sala de aula. Para Fontanella (2015), podem ser os responsáveis pela aproximação da escola à realidade do aluno que cresceu num universo digital, promovendo um maior envolvimento do discente com sua aprendizagem.

Neste cenário, a proposta desta pesquisa é o desenvolvimento de um objeto de aprendizagem voltado para a área do conhecimento de história. O projeto consiste em uma aplicação interativa para linha do tempo, na qual o usuário pode navegar através de fatos históricos visualizando claramente sua relação temporal. A interatividade da ferramenta permite, ainda, que o usuário navegue ajustando a faixa temporal, o que propicia uma boa navegação mesmo com uma quantidade grande de dados.

A história, área do conhecimento escolhida para a realização deste projeto, pode ser entendida, dentre várias outras definições, como um processo que abrange as mudanças temporais do mundo humano e social. Para tanto, como é comum a todas as ciências, foi necessário constituir uma racionalização metodológica como estratégia para a organização e a difusão do conhecimento produzido por ela.

Em se tratando da abordagem dessa ciência em sala de aula, Rüsen (2001) nos ajuda a entender a história como tempo significado e nos leva a inferir que o ensino escolar da história, tal como o conhecemos, é apenas uma das formas criadas por uma cultura específica - a ocidental moderna - para atender a necessidade da constituição de um passado em comum capaz de unificar os grupos sociais em um e garantindo a existência da ideia/sentimento de nação.

Neste sentido, é importante destacar que o ensino de história não é apenas um fenômeno da educação formal, constitui-se também, e essencialmente, como um fenômeno social, e como tal, busca estabelecer sentidos para o tempo vivenciado pela coletividade. Tais apontamentos sobre a história fazem-se necessários para demarcar que entendemos essa ciência/disciplina como algo que vai muito além de uma sequência de acontecimentos. Entretanto, quando pensamos em estratégias pedagógicas para aprendizagem em nível escolar, entendemos que organizar as informações em linha temporal auxilia no processo cognitivo de atribuição de significado, sentido, causalidade e lógica aos conteúdos que estão sendo estudados.

A área de visualização de dados é uma das áreas que subsidia esta pesquisa, trata-se de um campo de estudo relativamente novo, que pode ser uma resposta para a grande dificuldade de lidar com a quantidade enorme de informações que temos, hoje, disponibilizadas pela internet. De acordo com Aparicio e Costa (2015), a visualização de dados diminui a complexidade destes, tornando-os mais entendíveis e usáveis.

O desenvolvimento de uma aplicação interativa possibilita uma interação constante do estudante com a ferramenta, um aprendizado dinâmico e que explora a criatividade, como explicam Patten, Sánchez e Tangney (2006). Para Santos, Brennand e Soares (2016), aplicações interativas são potencializadoras de múltiplas inteligências, considerando a teoria das inteligências múltiplas (Gardner, 1995).

As aplicações existentes de linha do tempo não suprem a demanda que procuramos atender. Nossa proposta é uma ferramenta de linha do tempo fácil, intuitiva, interativa, com boa usabilidade, que realmente possa se tornar um objeto de aprendizagem no campo de conhecimento de história. Consultando a literatura, observamos o trabalho de Aguilar, Therón e García-Peñalvo (2009), que consiste de uma linha do tempo em espiral para suporte ao ensino à distância, complexa e de propósito diverso, mas que reforça o potencial de linhas do tempo. O trabalho de Monroy et al. (2002) propõe uma linha do tempo interativa para verificar 
modificações em documentos textuais, também de propósito diverso do nosso trabalho. Também citamos o trabalho de Lee, Ong e Messom (2011) no qual os pesquisadores desenvolveram uma ferramenta autoral em formato de linha do tempo com o objetivo de elaboração de livros didáticos.

A ferramenta existente que mais se aproxima de nossa proposta é a linha do tempo ${ }^{1}$ criada pela Biblioteca Digital Mundial, no inglês World Digital Library, uma biblioteca digital projetada pela Biblioteca do Congresso dos Estados Unidos da América e pela Organização das Nações Unidas para a Educação, a Ciência e a Cultura (UNESCO) em parceria com mais 31 outras instituições de vários países. Porém, essa ferramenta, ao mesmo tempo que é limitada em conteúdo, pois não permite que o usuário crie suas próprias linhas do tempo, também pode ser de complexa navegação.

Em nossa proposta, a ferramenta tem o diferencial de ser livre. É possível que o próprio usuário crie linhas do tempo específicas para estudar determinados assuntos e compartilhe-as, possibilitando diversidade de assuntos e, também, mais objetividade em cada linha do tempo através de uma interface minimalista.

\section{Material e Métodos}

A primeira etapa do trabalho foi a identificação da demanda por uma tecnologia que pudesse ser suporte para os estudos de história com recursos para navegação temporal. A demanda inicialmente foi identificada pelos discentes, participantes deste trabalho, durante seus estudos, juntamente com a docente de história também participante deste trabalho.

A partir de identificada a demanda, procurou-se formar uma equipe multidisciplinar para delimitar o escopo do problema e desenvolver a proposta. Fazem parte da equipe deste trabalho: dois discentes que na época cursavam o Curso Técnico em Informática Integrado ao Ensino Médio, uma professora de história e um professor de Computação.

Uma vez definido o escopo do projeto, então recorremos à literatura para buscar pesquisas anteriores e conceitos que pudessem dar suporte para nossa proposta. Concluiu-se que não havia uma ferramenta com as características que pudessem suprir a demanda identificada na área de história, porém identificamos conceitos que auxiliaram a conceber a proposta e a realizar a definição das tecnologias.

O ambiente web foi escolhido por sua flexibilidade e facilidade de compartilhamento de informações. Desta forma, a ferramenta estará disponível para todos que se conectarem pela internet. Como tecnologias para o desenvolvimento da aplicação foram usadas: HTML, CSS, JavaScript, PHP e MySQL.

Um quesito importante que norteou todo o desenvolvimento deste objeto de aprendizagem foi a usabilidade. Segundo Ferreira e Leite (2003), a usabilidade é o conceito que estabelece se determinado produto é manuseado e aprendido facilmente, não provoca erros operacionais e é eficiente para resolver as tarefas que se propõe. Desta forma, a usabilidade é um dos grandes diferenciais desta proposta.

Como validação para a ferramenta desenvolvida, criamos um formulário anônimo que estará continuamente disponível na ferramenta. Portanto, teremos um feedback contínuo dos usuários sobre problemas e sugestões para melhorar ainda mais os recursos.

\footnotetext{
${ }^{1}$ Disponível em: https://www.wdl.org/en/timelines
} 


\section{Análise de Resultados}

Yan et al. (2011) definem uma linha do tempo como um relato histórico de eventos variando em sequência cronológica. Eles afirmam ainda que uma das particularidades das linhas do tempo é que diferentemente das narrativas, que selecionam eventos em um contexto interpretativo, as linhas de tempo são mais conscientes do evento.

Aproveitando as características pedagógicas, docentes podem utilizar linhas do tempo para ajudar os estudantes a organizarem informações numa sequência cronológica para que possam entender melhor o crescimento, a mudança, os eventos recorrentes, as relações de causa-efeito e os principais eventos de importância histórica, social e científica.

Na Figura 1 e na Figura 2 ilustramos o uso da linha do tempo como ferramenta pedagógica para uma aula de história, supondo que o docente esteja trabalhando com conteúdos da Primeira Guerra Mundial com seus discentes.

Na Figura 1 navegamos pela linha do tempo interativa e selecionamos um evento importante na parte inferior e, então, foram exibidos seus detalhes na parte superior. $\mathrm{O}$ fato em questão é o assassinato do arquiduque Francisco Ferdinando, um dos marcos para o início da Primeira Guerra Mundial. A seta em vermelho identifica o evento selecionado.

Figura 1 - Linha do Tempo da Primeira Guerra Mundial, Evento: Assassinato de Francisco Ferdinando.

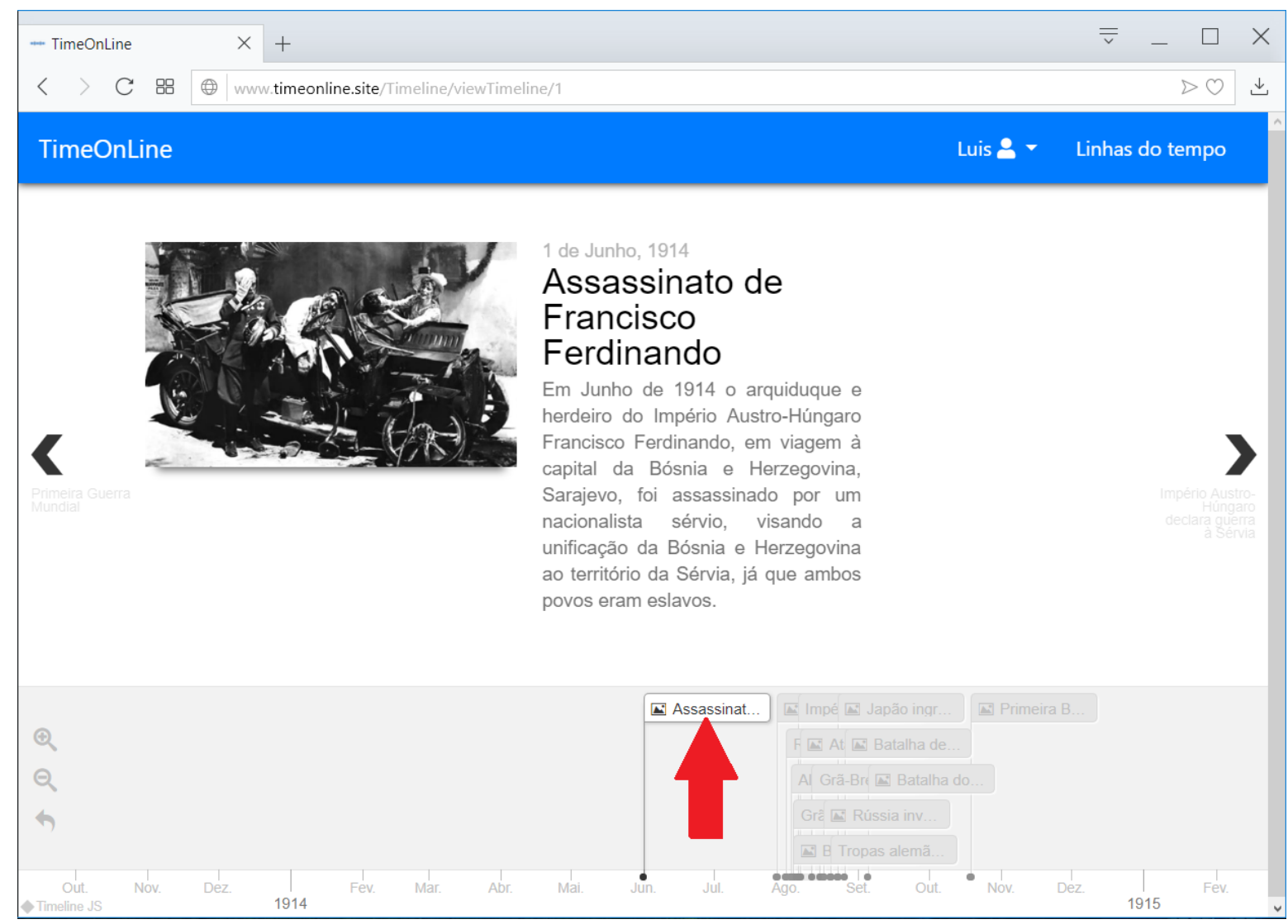

Fonte: Elaborado pelos autores, 2019. 
A Figura 2 mostra um evento subsequente, a declaração de guerra feita pelo Império Austro-Húngaro à Sérvia, que foi exibido ao se clicar no próximo item da linha do tempo, indicado também pela seta em vermelho. Desta forma, o professor pode mostrar claramente ao discente a disposição temporal dos acontecimentos e o discente, interativamente, pode observar outros eventos, verificar o posicionamento no tempo, analisar relações e compreender a noção de "fatos em cadeia", quando um acontecimento vira causa imediata de outro.

Figura 2 - Linha do Tempo da Primeira Guerra Mundial, Evento: Guerra à Sérvia.

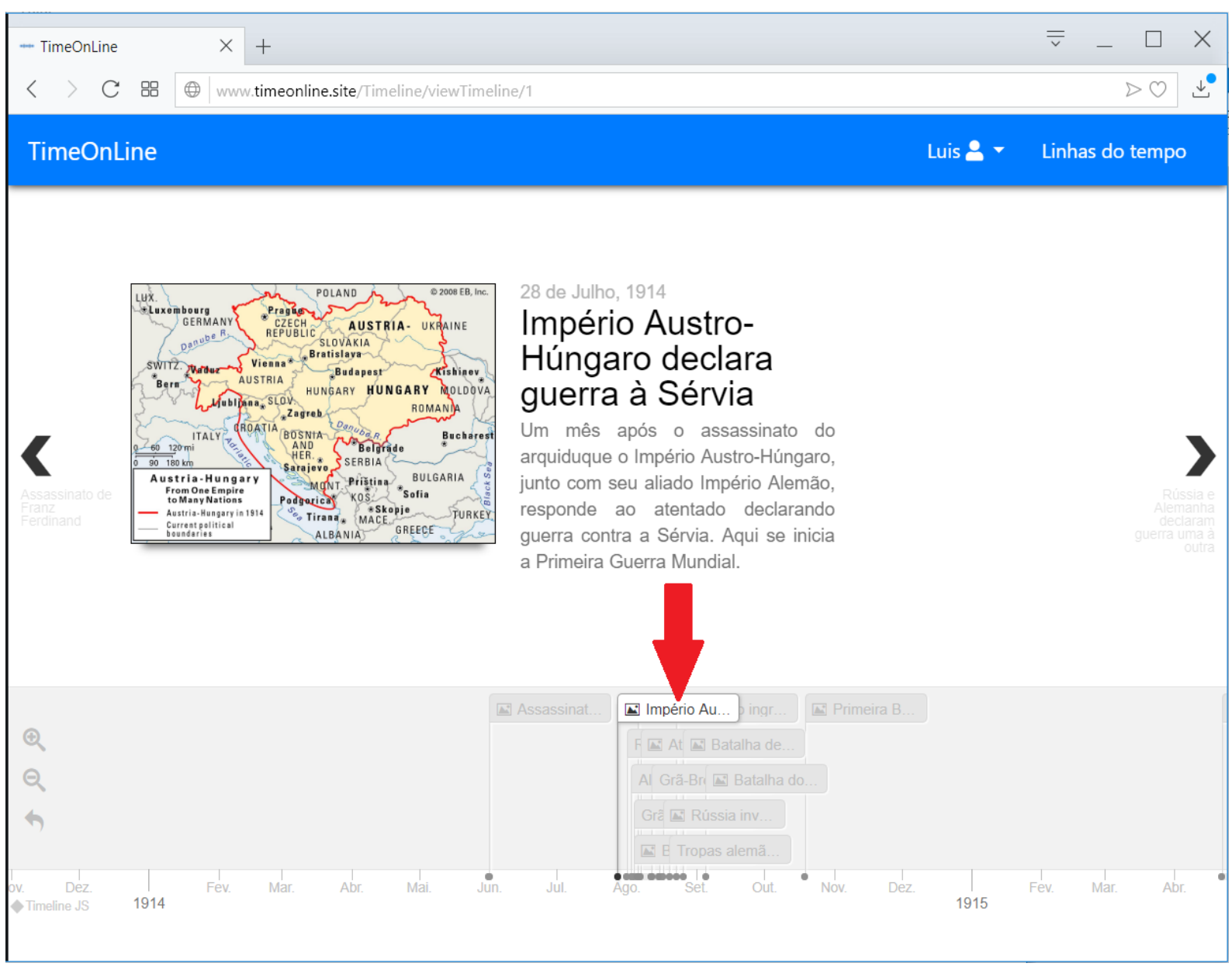

Fonte: Elaborado pelos autores, 2019.

A ferramenta demonstra um grande potencial didático devido a sua flexibilidade e dinamismo, pois o docente pode criar a linha do tempo que achar mais apropriada para as suas aulas. Neste sentido, dá liberdade para que ele possa utilizar este recurso de forma adaptada ao roteiro de aula que preparou, sem a necessidade de ficar preso a materiais prontos e inflexíveis. Além disso, ela conta com uma interface interativa, intuitiva e minimalista e, outro recurso importante, com a possibilidade de adicionar diversos tipos de mídia para se relacionar ao evento, como, por exemplo, imagens, vídeos, áudios, mapas e postagens de redes sociais.

A Figura 3 ilustra o início do processo de criação de uma linha do tempo. A seta em vermelho indica as ações que o criador da linha temporal pode realizar, como adicionar novos fatos e editar ou remover fatos já existentes. A inserção e edição de acontecimentos são 
realizadas a partir de um formulário que é disposto na tela assim que o botão referente à ação for selecionado.

Figura 3 - Edição de linhas do tempo.

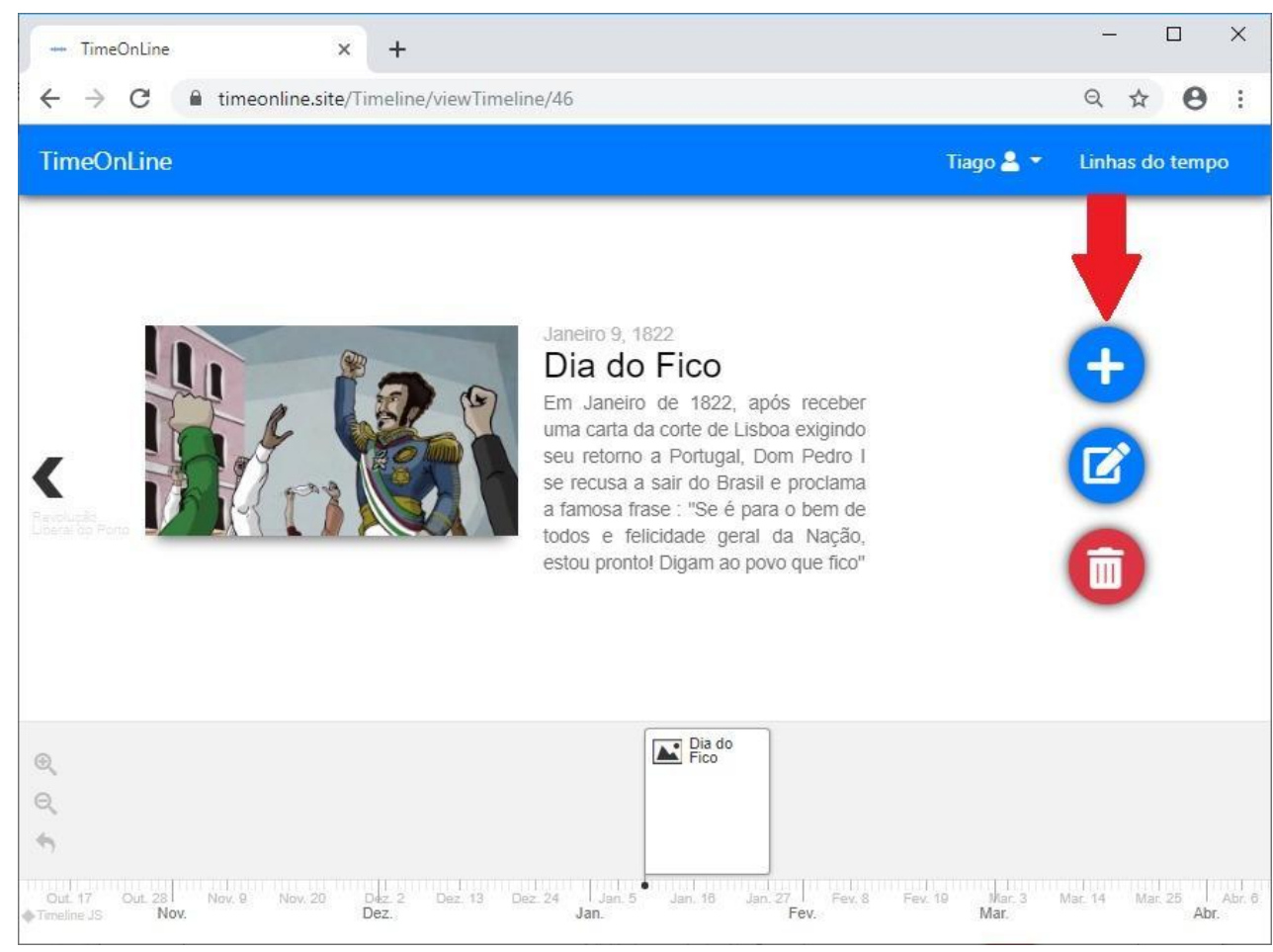

Fonte: Elaborado pelos autores, 2019.

Conforme explica Bates (2015), as mídias digitais estão cada vez mais integrando antigas mídias, como texto, áudio e imagens, e a novos componentes, como simulações, animações e interatividade. Uma mídia digital, ao incorporar esses vários componentes, tornase uma mídia "rica", possibilitando o desenvolvimento mais profundo de conhecimentos e habilidades. $\mathrm{O}$ uso de diferentes mídias também permite atingir mais discentes, abrangendo estilos e necessidades de aprendizagem diferentes. A internet é a grande responsável por permitir essa convergência de mídias.

Bates (2015) afirma ainda que mudanças no modelo de design básico das aulas são fundamentais para explorarmos as demandas da era digital e o potencial pleno das novas tecnologias na educação. Portanto, vemos nesta ferramenta pedagógica uma maneira de usar o potencial interativo de objetos de aprendizagem construídos com novas tecnologias, pois a interatividade, conforme explicam Patten, Sánchez e Tangney (2006), possibilita explorar o potencial criativo.

O desenvolvimento de uma aplicação tecnológica aproxima o processo de aprendizagem do nosso cotidiano digital, pois, hoje, a falta de conexão da escola com o mundo real (meio em que o discente vive) faz com que os alunos, muitas vezes, sintam-se desmotivados em aprender.

A ferramenta foi hospedada no endereço https://timeonline.site e está disponível para uso por qualquer usuário mediante um cadastro. Ela tem se mostrado muito flexível, acessível e prática, cumprindo os objetivos da proposta. Pretende-se disseminar o uso da ferramenta entre docentes e discentes para podermos coletar mais dados a fim de validação e melhorias. 


\section{Conclusões}

No mundo contemporâneo, as tecnologias de informação são ferramentas importantes e cada vez mais essenciais no cotidiano dos indivíduos. Neste sentido, é possível afirmar que a exclusão digital tem forte impacto na inserção social. Assim, torna-se imprescindível o uso das tecnologias de informação e de comunicação nas escolas, tanto para democratizar seu acesso quanto para incluir o máximo de pessoas no mundo digital.

A presente proposta trouxe algumas contribuições, entre elas suprir a demanda por objetos de aprendizagem na área de história, uma área do conhecimento ainda com poucas soluções tecnológicas como apoio no processo de aprendizagem.

Outra importante contribuição é o desenvolvimento de uma solução tecnológica aplicada ao ensino, um objeto de aprendizagem, que colabora para diminuir a distância entre a realidade tecnológica que vivenciamos no mundo e a realidade escolar, que se apropria da tecnologia ainda a passos lentos.

Ressaltamos também que a ferramenta demonstra que soluções de visualização de dados podem trazer muitos benefícios, auxiliando a lidar com o volume rico e crescente de dados que temos disponíveis através da internet.

Ao disponibilizar o acesso à ferramenta de forma gratuita, esperamos que ela possa contribuir com o aprendizado de muitos discentes e receber feedbacks para avaliações de melhorias. Como proposta de trabalho futuro, sugerimos estudos que avaliem grupos de estudantes fazendo uso desta ferramenta em práticas escolares.

\section{Referências}

AGUIAR, E. V. B.; FLORES, M. L. P. Objetos de Aprendizagem: Conceitos Básicos. In: TAROUCO et al. (org.). Objetos de Aprendizagem: Teoria e prática. Porto Alegre: CINTED/UFRGS; Evangraf 2014. p. 12-28.

AGUILAR, D. A. G.; THERÓN, R.; GARCÍA-PEÑALVO, F. Semantic Spiral Timelines Used as Support for e-Learning. Journal of Universal Computer Science, v. 15, p. 1526$1545,2009$.

APARICIO, M.; COSTA, C. J. Data Visualization. Communication Design Quarterly Review, v. 3, n. 1, p. 7-11, 2015.

ARROYO, M. G. Ofício de mestre: imagens e auto-imagens. Petrópolis: Vozes, 2000.

BATES, A. W. Teaching in a Digital Age: Guidelines for Designing Teaching and Learning Vancouver BC: Tony Bates Associates, 2015.

BRASIL. Ministério da Educação. Secretaria da Educação Básica. Base Nacional Comum Curricular. Brasília, DF, 2017. Disponível em: 〈http://basenacionalcomum.mec.gov.br〉. Acesso em: 10 nov. 2018.

CÂNDIDO et al. Aproximação das TICS na Educação: Possibilidade e Considerações.

Revista Inovaeduc, n.4. Faculdade de Educação da Universidade Estadual de Campinas (FEUnicamp), 2018. Disponível em: <http://www.lantec.fe.unicamp.br/inovaeduc/wpcontent/uploads/2018/n4.art2.pdf>. Acesso em: 05 nov. 2018. 
FERREIRA, S. B. L.; LEITE, J. C. S. P. Avaliação da usabilidade em sistemas de informação: o caso do Sistema Submarino. Revista de Administração Contemporânea, Curitiba, v. 7, n. 2, p. 115-136. Disponível em: $<$ http://www.scielo.br/scielo.php?script=sci_arttext\&pid=S141565552003000200007\&lng=en\&nrm=iso>. Acesso em: 08 nov. 2018.

FONTANELlA, A. C. Uso de Objeto de Aprendizagem na Motivação e Aprendizagem Matemática. Trabalho de Conclusão de Curso Especialista em Mídias na Educação (Especialização). Universidade Federal do Rio Grande do Sul, 2015.

FREITAS, N. M. C. Objetos de aprendizagem para o ensino da história: uma busca na Web. Trabalho de Conclusão de Curso Especialista em Tecnologias da Informação e Aplicadas à Educação (Especialização). Universidade Federal de Santa Maria, 2010. Disponível em: <https://repositorio.ufsm.br/handle/1/864>. Acesso em: 07 nov. 2018.

GARDNER, H. Inteligências múltiplas: a teoria na prática. Porto Alegre: Artes Médicas, 1995.

HELLER, A. Uma Teoria da História. Rio de Janeiro: Civilização Brasileira, 1993.

LEE, H. J. J.; ONG, T. J.; MESSOM, C. H. Timeline-based authoring tool for e-Textbook: Bringing the "mind-mapped learning model". Proceedings: Frontiers in Education Conference, FIE, p. S1E-1- S1E-5, 2011.

LÉVY, P. As tecnologias da inteligência: o futuro do pensamento na era da informática. 2. ed. São Paulo: Editora 34, 1993.

MONROY et al. Interactive Timeline Viewer (ItLv): A Tool to Visualize Variants among Documents. In: Börner K., Chen C. (org.). Visual Interfaces to Digital Libraries. Lecture Notes in Computer Science, v. 2539. Springer, Berlin, Heidelberg. 2002.

MORAN, J. M. A educação que desejamos: novos desafios e como chegar lá. 2. ed. Campinas, SP: Papirus, 2007.

PATTEN, B.; SÁNCHEZ, I. A.; TANGNEY, B. Designing collaborative, constructionist and contextual applications for handheld devices. Computers \& Education. v. 46, p. 294-308, 2006.

RÜSEN, J. Razão Histórica. Brasília: EdUNb, 2001.

SANTOS, R. A.; BRENNAND, E. G. G.; SOARES, I. M. Aplicações interativas como potencializadoras de múltiplas inteligências. ETD - Educação Temática Digital, Campinas, v. 18 , n. $2,2016$.

TAROUCO, L. M. R.; FABRE, M. C. J. M.; TAMASIUNAS, F. R. Reusabilidade de objetos educacionais. Revista Renote, Porto Alegre, v. 1, n. 1, p. 1-11, 2003. Disponível em:

<http://seer.ufrgs.br/index.php/renote/article/view/13628/7697>. Acesso em: 23 out. 2019. 
YAN et al. Evolutionary timeline summarization: a balanced optimization framework via iterative substitution. In: Proceedings of the 34th international ACM SIGIR conference on Research and development in Information Retrieval (SIGIR '11). ACM, New York, NY, USA, 211, p. 745-754.

WILEY, D. A. Connecting Learning Objects to Instructional Design Theory:

A Definition, a Metaphor, and a Taxonomy. In: WILEY, D. A (org.). Instructional Use of Learning Objects. Bloomington, Indiana: Agency for Instructional Technology, 2001.

ZUIN, A. A. S. O Plano Nacional de Educação e as Tecnologias da Informação e Comunicação, Revista Educação e Sociedade, Campinas, v. 31, n. 112, p. 961-980, 2010. Disponível em: <http://www.scielo.br/pdf/es/v31n112/16.pdf> Acesso em: 06 nov. 2018.

Recebido em julho de 2019.

Aprovado em outubro de 2019. 